\title{
Simulation and Improved Channel Assignment by Simulated Annealing of a Wireless Mesh Network using Dynamic Spectrum Access
}

\author{
Natasha Zlobinsky \\ natzlob@gmail.com \\ University of Cape Town \\ Cape Town, South Africa \\ Amit K. Mishra \\ akmishra@ieee.org \\ University of Cape Town \\ Cape Town, South Africa
}

\author{
David L. Johnson \\ david.lloyd.johnson@gmail.com \\ University of Cape Town \\ Cape Town, South Africa
}

\author{
Albert A. Lysko* \\ alysko@csir.co.za \\ Council for Scientific and Industrial Research \\ Pretoria, South Africa
}

\begin{abstract}
This work tackles a new angle to the Channel Assignment (CA) problem, which has otherwise been fairly widely studied for allocating channels optimally to access points and ad-hoc network nodes. Wireless Mesh Networks (WMNs) using Dynamic Spectrum Access (DSA), such as Television White Spaces (TVWS), create new avenues for research due to the additional constraints and complexity. For the production of controlled and repeatable experiments and design of CA algorithms without the drawbacks and difficulties of real hardware, we use Network Simulator 3 (ns3). In this paper we address the construction of an experimental setup in ns3 for evaluating CA algorithms in a DSA WMN environment. Additionally, we propose a solution to the CA problem in this scenario using Simulated Annealing. We simulate TVWS device operation by adding TVWS channels (this can be extended to include any new DSA bands), provide a framework for multi-radio multi-channel WMN experiments, and present and analyse the performance of a CA algorithm. Results show that the proposed algorithm provides channel assignments with much improved performance (120\%-755\% better) over random channel assignments.
\end{abstract}

\section{CCS CONCEPTS}

- Networks $\rightarrow$ Network resources allocation; Network simulations; Network performance modeling.

\section{KEYWORDS}

Network Simulator 3; NS3; simulation; modeling; wireless mesh networks; WMN; TVWS; DSA; Cognitive Radio; optimisation; channel assignment; Simulated Annealing; unlicensed spectrum

\footnotetext{
*Also with University of Cape Town.

Permission to make digital or hard copies of all or part of this work for personal or classroom use is granted without fee provided that copies are not made or distributed for profit or commercial advantage and that copies bear this notice and the full citation on the first page. Copyrights for components of this work owned by others than the author(s) must be honored. Abstracting with credit is permitted. To copy otherwise, or republish, to post on servers or to redistribute to lists, requires prior specific permission and/or a fee. Request permissions from permissions@acm.org.

MobiWac '21, November 22-26, 2021, Aliante, Spain.

(C) 2021 Copyright held by the owner/author(s). Publication rights licensed to ACM ACM ISBN 978-1-4503-9079-8/21/11...\$15.00

https://doi.org/10.1145/3479241.3486696
}

\section{ACM Reference Format:}

Natasha Zlobinsky, David L. Johnson, Amit K. Mishra, and Albert A. Lysko. 2021. Simulation and Improved Channel Assignment by Simulated Annealing of a Wireless Mesh Network using Dynamic Spectrum Access. In Proceedings of the 19th ACM International Symposium on Mobility Management (MobiWac '21), November 22-26, 2021, Aliante, Spain. ACM, New York, NY, USA, 10 pages. https://doi.org/10.1145/3479241.3486696

\section{INTRODUCTION}

The Channel Assignment (CA) problem in wireless mesh networks (WMNs) continues to be a popular area of research (see, for example, $[3,6,8,19,27])$. The topic is continually evolving as new applications and complexities related to new technologies and spectrum management approaches emerge. For example, the problem can now be extended to consider channel allocation in WMNs using Dynamic Spectrum Access (DSA) or Cognitive Radio (CR) technologies and bands, such as Television White Spaces (TVWS), $6 \mathrm{GHz}$ band for Wi-Fi 6E, and Citizens Broadband Radio Service (CBRS).

DSA using spectrum databases has recently gained renewed momentum with Spectrum Access System testing for CBRS completed in June 2019 [1], and Automated Frequency Selection being specified for Wi-Fi 6E [2]. In April 2020, the Federal Communications Commission (FCC) voted unanimously to open up all of the $6 \mathrm{GHz}$ band for this and other unlicensed use [22]. One of the alternative frequency bands that still has much potential, particularly in the developing world and rural areas, is TVWS. It is currently difficult to get hold of affordable hardware for testing TVWS and the current hardware does not support mesh mode. There is also still a number of research questions related to its use in WMNs, which have not been addressed in the literature. While the work presented in this paper is applicable to all DSA bands, we concentrate on TVWS.

Since the hardware to incorporate these new spectral bands may not be available, or is prohibitively costly to acquire for experimentation purposes, simulation becomes a critical tool for researchers wishing to develop new CA algorithms or to evaluate the performance of existing algorithms. Even if the hardware exists, getting a sufficient number of nodes to evaluate the performance of algorithms at scale may not be feasible, and producing reproducible and controlled experiments may not be possible outside of a simulation environment. Network Simulator 3 (ns3) is one such simulation 
environment. It was chosen for our work because it has a robust community, is widely used, has thorough documentation, and provides most elements required for setting up CA experiments in WMNs. While most of the elements we required are available, setting up simulations with all the necessary aspects for CA in WMNs with nodes having multiple radio interfaces, different traffic flows, multiple channels, and dynamic channel changes is challenging to configure in ns3. For this reason, we have created a framework for testing new CA algorithms and heuristics in ns3 that is easy to adapt and implement. There is also currently almost no support for alternative frequency bands and DSA in ns 3 and it does not provide any models for White Space Devices (WSDs) or ways to simulate the behaviour of WSDs.

In this work we also aim to address the open research area of assigning channels optimally in a WMN that uses the TVWS spectrum band in the presence of external interference, such that interference is minimised and Signal to Interference and Noise Ratio $(S I N R)$ is maximised. We use a Simulated Annealing algorithm to this end, and evaluate its performance.

This paper is structured as follows: In Section 2 we provide background to TVWS, the CA problem and meta-heuristic algorithms relative to our problem. Section 3 documents some of the most notable related work. The problem is formally described in Section 4 . We then present the multi-radio multi-channel WMN simulation framework developed in Section 5.1. The way we enabled the simulation of WSDs is presented in Section 5.2, interference modelling is presented in Section 5.3, Simulated Annealing in Section 5.4 and results are presented and discussed in Section 6. Before concluding we discuss practical issues and possible future developments on the topic in Sections 7 and 8.

\section{BACKGROUND}

\subsection{TVWS overview}

TVWS is the term for the unused portion of the spectrum traditionally allocated for use by terrestrial broadcast television. Owing to the way that television transmitters are deployed and frequencies are allocated, there are large portions of the spectrum that are unused in certain locations, particularly outside of cities. TVWS saw increased interest in the research community since over a decade ago when all around the world much of the analogue television systems were being switched to digital TV, opening up even more of the spectrum for unlicensed use by other services [15, 24, 31]. The lower frequencies of the TVWS band mean that TVWS has propagation characteristics that far out-class those of the traditional Wi-Fi spectrum. It has a much longer range and the ability to penetrate a large variety of obstructions, allowing operation even in non-line-of-sight scenarios. However, this ability also varies by TV channel as it is proportional to the wavelength. TVWS is ideal for delivering connectivity to traditionally under-served communities in rural or outlying areas that are difficult to reach either by fibre or are being neglected by mobile providers owing to the relative lack of economic drive to deliver connectivity to these areas.

On the other hand, the better propagation properties in TVWS could cause more interference between devices than higher frequency bands with lesser reach, and so is not so suitable to highdensity deployments. Another issue that arises in the UHF spectrum that is not as prevalent in other bands is the wide dynamic range and differences in propagation characteristics in different channels within the band.

A WSD accesses the TVWS without a licence and is called a secondary user (SU) for this reason. It is required by most TVWS regulations (as well as other DSA bands) that the unlicensed device wishing to access the spectrum band obtain a list of available and allowed channels from a Geolocation Spectrum Database (GLSD) in order to operate. This way, interference with the primary users (TV transmitters) is prevented [9, 29]. This means that in different locations the allowed channels for SUs may differ. However, GLSD methods are pretty ham-fisted and do not ensure high channel utilisation efficiency, as the error margins are often very large. The GLSD also cannot inform a WSD of other SUs in the area that may not be a part of its network but are also using the TVWS spectrum. This means that WSDs that wish to use TVWS spectrum also need to be able to perform spectrum sensing to determine what channels are truly free for use and which have too much interference from other SUs, or from TV transmitters with leaky spectral masks, or because of poor receive filtering.

These issues combined raise the question of how best a WSD can choose a channel on which to operate. The question becomes even more complicated when the WSD in question is also a WMN node that is part of a large network of nodes that all use the TVWS spectrum and which may cover several geographic locations with different allowed channels and different interference sources in their vicinity. A first step towards answering this research question is to be able to develop a simulation that can model all the aforementioned objects and phenomena. The interference aspects are of particular importance. This is what we have tried to achieve in this work.

\subsection{Channel Assignment problem in Wireless Mesh Networks}

Since different channels may be optimal for use by different nodes in a WMN and different channels may experience different levels of external interference and utilisation, the problem of assigning channels optimally is an important and difficult one in wireless networks. The CA problem is well known to be NP-hard since it is in essence a graph-colouring problem [12]. Despite considerable attention to the question in the literature there is currently no universally good solution to this CA problem in WMNs, and substantial opportunities for further research and improvements exist in the case of DSA and CR.

A good CA must minimise the interference experienced within the network and externally, maximise $S I N R$, maintain connectivity along necessary paths between nodes, and improve overall network capacity and performance. We must consider that an optimal CA changes according to the routes followed by packets (selected by the routing algorithm), which links are used, and where the bottleneck links are in the network. Conversely, routing depends on the capacity of links, which is dependent on the CA. Hence, routing and Channel Assignment are very much interlinked. However, every new packet that is offered to the network may need new routing and so is a very dynamic process, while changing channels is usually a slower process since it takes physically on the order of seconds for 
a Network Interface Card (NIC) to switch its channel and reconnect the link for all current hardware. Hence, routing must be taken into account, but the CA cannot only be applicable to a snapshot of routes and must be a more global longer term solution.

A CA algorithm that takes into account parameters that change more quickly, e.g. link quality, traffic conditions and routes, is more adaptive to changing conditions in the network and may be optimal more of the time. However, it would result in loss of connectivity more often. Overall, this degrades network performance. A rapidly changing CA would also cause the routing to have to re-adapt often and may result in a race condition between routing and $\mathrm{CA}$, causing the network to become unstable. An optimal CA would have to take spectrum measurements over time covering a variety of routing configurations and determine over that time what are the most used or most congested links, or do topology discovery, and use this information as an input to the CA algorithm.

$\mathrm{CA}$ algorithms might increase overhead from the communication required to distribute channel information, exchange other decisionmaking information and update the state of the network. We do not consider overhead in this work.

\subsection{Meta-heuristic algorithms for finding near-optimal solutions}

Meta-heuristic algorithms find either approximate solutions to optimisation problems or solutions to not all instances of a problem [18]. They are used for complex, usually NP-hard or NP-complete, problems. Two classes of meta-heuristic strategy are divide-and-conquer and iterative improvement. In general, an iterative procedure would be to start with the system in a known state with an initial possible solution, and then effect changes to the system step by step, searching through the solution space to improve on that first solution. Several such meta-heuristics algorithms for approximate optimisation exist. Examples are Genetic Algorithm (GA), Simulated Annealing and Tabu search. These meta-heuristics are used for NP-hard or NP-complete problems because there is no way to find an exact solution within a bounded reasonable time (unless $P=N P$ ).

We have used Simulated Annealing (SA) in this work. SA is an iterative probabilistic search heuristic used in optimisation problems with complex search spaces. It is based on, and analogous to, the physical process of annealing in statistical mechanics, whereby atoms are cooled in a specific slow way until reaching the state of minimum energy [16]. The algorithm is outlined in Figure 1. It starts with the system in a certain configuration or state, i.e. the starting solution, and we must find the "energy", which is the value of the objective function or cost of that solution at that iteration. From there, a new neighbour solution is generated and its cost value computed and compared to the first. The new solution is then either accepted or rejected based on whether it has a lower cost than the first solution and according to the temperature parameter.

The temperature parameter is related to how likely we are to choose a worse solution than the current one to ensure the algorithm does not get stuck in a local minimum. The temperature must initially be set to a certain higher value and is decreased every iteration according to a defined cooling function, the choice of which is up to the implementer. The process of generating a new neighbour solution and accepting or rejecting the solution continues until the defined number of iterations or acceptable running time is reached and an acceptably low solution has been settled on. The aim is always to find the lowest "energy" (cost) configuration by the end. As the number of iterations increases, so the probability of finding the true optimal solution increases. However, there are certain tests or rules-of-thumb that can be followed to estimate the convergence time, e.g. the Geweke test [11].

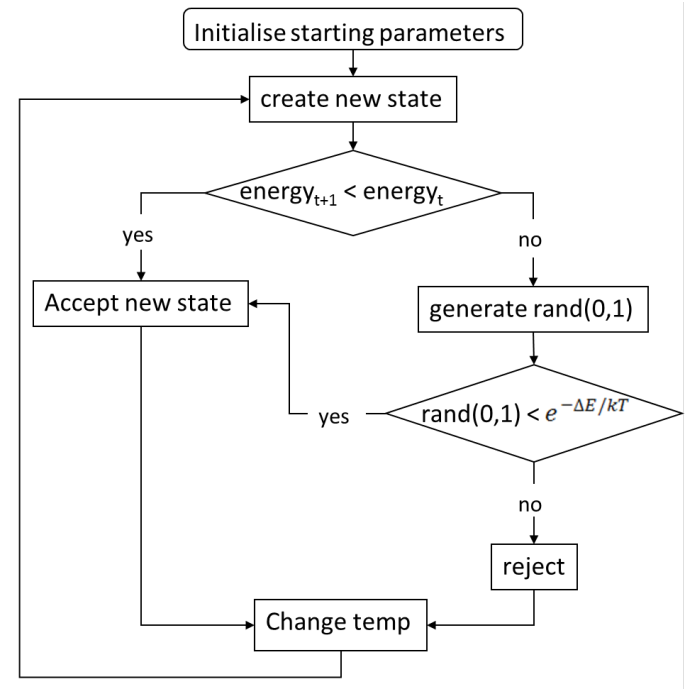

Figure 1: Simulated Annealing (temp=temperature, rand $(0,1)$ is a sample of uniformly distributed random number $\sim U(0,1)$ and $\Delta E, k$ and $T$ are defined in Section 5.4)

\section{RELATED WORK}

This section is not, by any means, a complete account of all multiradio multi-channel (MRMC) CA literature, but merely highlights some of the most relevant to our work. Much work has been done on CA, including on MRMC scenarios e.g. [5, 7, 17, 23, 28, 30]. There has even been work that uses Simulated Annealing for the CA problem in WMNs [6]. However, we are not aware of any prior work that addresses the problem in the case of DSA. In this work we also focus on the details of the simulation setup required for addressing the problem. Our formulation also allows easy extension to the case of adjacent channel interference, and channels further away.

Raniwala et al. [23] present two of the first MRMC CAs that do not require alterations to the 802.11 MAC layer: a centralised algorithm that integrates routing, and another load-aware algorithm that takes into account channel capacity. The goal of this algorithm is to maximise goodput and does not take either internal or external interference level into account. Subramanian et al. [30] present a Tabu search-based algorithm that aims to minimise interference. Interference is only quantified as the number of conflicts, and so is modelled as a binary phenomenon, instead of the real interference power. Ko et al. [17] present a distributed self-stabilising CA. Their formulation allows for overlapping channels, and thus adjacent 
channel interference, but they simply model this interference as a linearly decreasing function with spectral distance. Shortcomings in this work are that they prove that the network will stabilise within a finite number of channel changes, but there is no bound on that number so its practical application is limited, and sophisticated coordination is required between nodes for this method to work.

In [28], the authors present centralised load-based CAs. One is constructed by Lagrangian relaxation of the NP-hard Integer Linear Programming problem formulation, and the other uses a GA. They also present a hill-climbing-based distributed algorithm. In their work, the link with routing is incomplete and interference is once again considered binary. A discrete particle swarm solution for minimizing co-channel interference is presented by Cheng et al. [7]. This paper also considers interference as binary and simply minimises the number of collisions. Another GA-based solution is put forward by Balusu et al. They consider a multi-cast tree WMN topology and optimise on channel conflict [5]. Finally, a Simulated Annealing solution is also presented by Chen et al. [6], where interference is considered in terms of conflict but is weighted by the traffic on the links, which is assumed to be known and stable during the period of CA. This is not a realistic assumption. None of the aforementioned works address external interference, DSA or a realistic interference model as we do in this paper.

Amiri-Nezhad et al. detail some aspects of simulating a MRMC mesh network in ns3 for the purposes of developing a CA algorithm [4]. This work is based on a decade-old version of ns3: version 3.9, released in 2011, and so does not consider all the subsequent developments to ns3. It also has limitations such as fixing the data rate and using static routing. An interference emulator is introduced to simulate external interference, but it only models channel usage in binary terms as either busy or idle, equating channel utilisation in terms of busy time with interference. This work was developed before the existence of the SpectrumPhy class in ns 3 that allows interference between different networks and device types. Hence, it does not consider the actual interference power of external interfering signals, such as TVWS.

On the other hand, Shen et al. consider DSA rendezvous channel selection in CR Networks [26]. Nodes must find a (not necessarily optimal) channel that is jointly available to pairs or groups of nodes. Variation in the interference experienced by different nodes from external sources is taken into account, but internal interference is not. The simulation results presented are only for groups of two nodes and WMN aspects are not taken into account, so its use in a WMN is limited. Network time synchronisation is required, which is very difficult to achieve in practice.

In considering the literature, in addition to providing an updated and simpler implementation of a MRMC WMN for developing CA algorithms, an important contribution that we add is the combination of DSA aspects with WMN and a more realistic model of interference.

\section{PROBLEM FORMULATION}

We assume a known WMN topology where the most important active links are known, forming a graph $G=(V, E)$ where $V$ is the set of nodes (vertices) and edges $E$ are the set of active links between nodes. A link is defined as a pair of radio interfaces between which traffic could potentially flow directly if tuned to the same channel. Each edge $e \in E$ is tuned to a particular channel at any time, i.e. $e \mapsto c \in C$, where $C$ is the full set of considered allowed channels for the whole network. This would be the intersecting sets of channels allowed in different locations of the network according to the GLSD. A channel is specified by a channel number. There might also be other transmitting devices external to the considered WMN that can influence the reception of nodes in $G$ if they are transmitting with power in the same channel that one of the links $E$ is tuned to.

Connectivity graph $G$ maps to a conflict graph $G_{c}=\left(V_{c}, E_{c}\right)$ where the vertices of the conflict graph are the edges in $G$ and an edge $E_{c}$ exists between two vertices in $V_{c}$ if the two links interfere; that is, if there is a transmission in link 2 that causes power to leak into, or be transmitted in, the channel on which link 1 is operating. This can occur if two links are tuned to the same channel, or if links are tuned to different channels while the spectrum mask of the transmitter node is wide and there is power leaking into the channel on which link 1 is operating, or receive filtering is poor. We also add vertices and edges representing outside sources of interference, but note that these are fixed as their channels cannot be switched and their transmit power cannot be controlled. We can model this as a weighted conflict graph denoted $\left\langle G_{c}\left(V_{c}, E_{c}\right), w\right\rangle$, where the weight $w$ represents the level of interference or the interference power per channel. Considering this weighted conflict graph, we aim to minimise the conflict but maximise the wanted signal power received by each node, maintaining connectivity in $G$. The optimisation objective is thus to find the channel assignment $A$, which is a mapping of $E \mapsto C$ that maximises the total SINR, i.e.

$$
\begin{aligned}
\max _{A=E \mapsto C} \sum_{v \in V} \frac{P_{\text {wanted, }}(A)}{\sum_{i \in I} P_{i}(A)+N} & =\min _{A} \sum_{v \in V} \frac{\sum_{i \in I} P_{i}(A)+N}{P_{\text {wanted }, v}(A)} \\
& =\min _{A} \sum_{v \in V} \frac{\sum_{x \in V \backslash u, v} P_{v, x}(A)+N}{P_{v, u}(A)} \\
& =\min _{A} \sum_{v \in V} 1 / \operatorname{SINR}_{v}(A)
\end{aligned}
$$

over all possible channel assignments $A$, where:

$N$ is the noise floor, which in ns 3 is modeled as the thermal noise $\times$ noise figure

$P_{v, u}$ is the power received at node $v$ from transmitting node $u$

$P_{i}$ is interfering power received at node $v$ from an interfering transmission $i$. A transmitting node is considered interfering with $v$ if it is in the set of nodes $V$ minus the node $u$, the node transmitting a wanted signal to $v$ (and minus $v$ itself). We only consider there to be one wanted receive signal at a time. We can find $P_{i}$ using equation (2):

$$
\begin{aligned}
P_{i} & =\int_{f_{c}-w i d t h_{c} / 2}^{f_{c}+w d t h_{c} / 2} p\left(f_{b}\right) S_{t}\left(f_{b}\right) S_{r}\left(f_{c}\right) \mathrm{d} f \\
& =P_{v, x}(A) \quad \forall x \in V \backslash u, v
\end{aligned}
$$

where

$p\left(f_{b}\right)$ is the power spectral density of the interfering signal at the central frequency of channel $b$ in which the interfering node is transmitting. (It is possible that $b=c$ )

$S_{t}\left(f_{b}\right)$ is the spectral mask of the transmitter (interfering signal) centred at the central frequency of channel $b$ 
$S_{r}\left(f_{c}\right)$ is the receive filter's frequency response, which is tuned to channel $c$ and all is integrated over the width of the considered channel $c$.

This formulation allows for extension to the case of adjacent channel interference, or interference between any two channels, which is kept as future work.

Each transmitted signal is subject to propagation loss as well as frequency-selective fading. As usual, the received signal power at node $v$ from node $u$ 's transmitted power $P_{v, u}$ (in W) (before receive filtering) is related by the propagation loss $L$ according to the chosen loss model. We will consider the basic Friis free space propagation loss model of equation (3). This also implies that we assume an isotropic antenna model. It must be noted, however, that the applicability of our method is not limited to these models.

$$
P_{v, u}=P_{u} \frac{G_{v} G_{u} \lambda^{2}}{(4 \pi d)^{2}}=\frac{P_{u}}{L_{v, u}}
$$

where

$G_{u}$ is the transmission gain of node $u$ (unitless)

$G_{v}$ is the receive gain of node $v$ (unitless)

$\lambda$ is the wavelength (in $\mathrm{m}$ )

$d$ is the distance between the nodes $u$ and $v$ (in m)

$L_{v, u}$ is the propagation loss experienced between nodes $v$ and $u$

\section{MODELLING AND SIMULATION FRAMEWORK}

The goal of the research work for which simulations are to be done is CA in a WMN using DSA, and to do the CA via a metaheuristic optimisation algorithm to improve the performance. We have developed a framework in ns3 for all three parts of this goal. The code can be found at our ns3 fork for replication and reuse.

\subsection{The multi-radio multi-channel WMN simulation module}

A specific channel assignment is defined as a mapping of links to channels for all relevant/critical links in the network. In a real-world implementation, this set of links may be chosen to be the links that are found over time to have the most traffic or to be bottlenecks in the network. One of the nodes in the network might also be a gateway to the Internet (and the GLSD), and there might be a set of links that are often used to direct traffic through to the gateway and are thus the most constrained and the most in need of proper CA. Topology discovery or the method by which these critical links would be identified in a real network is out of the scope of this work.

In this simulation, we set up each node with two interfaces (radios), where one is a "server" of User Datagram Protocol (UDP) traffic, and the other a "client". In the ns3 implementation of all network protocols, there is a server-client model linking sent data units to receivers, even if the actual protocol does not have a server-client architecture, as in the case of UDP. This server-client designation is only used for generating traffic in the simulation and is done without loss of generality of the algorithm or experiments presented in this work. A link is represented by a pair of node IDs, where the first in the pair is the server interface of the first node and the second is the client interface of the second node. A vector of links represents the topology of the part of the network requiring optimal channel allocation, i.e. the critical links. We generate three different topologies for each considered WMN size and generate Constant Bitrate (CBR) traffic between the node pairs so as to saturate the links. While the network is multi-hop, channels are assigned per link and not per source-destination node pair, which might have several hops in between. Hence, we only consider assigning channels to links and do not need to be explicitly concerned with routing or the multi-hop paths, as this is taken care of by defining the links.

We add user-defined modules to ns3 for each of the two separate aspects of the program: the mesh simulation and the optimisation algorithm. We add a new mesh-sim module for the automation of simulation of a WMN with nodes having multiple interfaces on different channels, and with external interference. In this module we use a SpectrumPhy and MultiModelSpectrumChannel to ensure signals of different standards can coexist and interact on the same channel. The simulation is set up so that different loss models or parameters can be added to different channels, to model the behaviour of TVWS where different channels have different characteristics. However, the same channel model has been used for the experiments whose results are presented in Section 6, since our presented method works independently of the model chosen. The nodes are static and placed in a grid, although other positioning can easily be configured. Optimized Link State Routing (OLSR) is applied. Mesh nodes are defined with a configurable number of interfaces but set to two for the purposes of the results presented. The CBR traffic generated is made of fixed sized packets with a packet interval small enough to saturate the link.

Parameters passed to the mesh-sim program are a vector of "links", each defined as a std: : pair of integers specifying the node IDs of the two nodes, and the vector is the set of all such links. Also to be passed in to the mesh-sim program is a reference to a mapping of links to channel numbers, which is the actual channel assignment. A function then assigns each interface to its relevant channel. This function to set channels can be called multiple times in one simulation, so as to change channels mid-simulation.

\subsection{Modelling and simulation of the TVWS band}

In order to use the TVWS band in a Wi-Fi style MWN, we have built on top of the existing machinery for Wi-Fi in ns3, using as a basis the Wi-Fi PHY, but shifting the frequency and changing channel width to match the TVWS channels defined by the Independent Communications Authority of South Africa (ICASA) [13]. We have used the channel definitions prescribed by ICASA as this is the relevant regulation we need to abide by in our reallife test bed, which is being simulated in this work. However, the band and bandwidth are similar or overlapping in other countries (e.g. Ofcom in the United Kingdom) and the definitions we have added can be easily adjusted to follow definitions of other regulatory bodies. This "down-conversion" of Wi-Fi is similar to the way some real TVWS chips (e.g. Doodle labs DL509-78 Broadband Radio Transceiver for the 470-784 MHz TV band) functioned, which down-converted IEEE $802.11 \mathrm{a} / \mathrm{b} / \mathrm{g}$ to the relevant frequencies and channel widths for TVWS. The TVWS channels that are defined by ICASA are channels 21 to 48 from $470 \mathrm{MHz}-694 \mathrm{MHz}$ and 
have $8 \mathrm{MHz}$ channel widths. We declare a new standard in ns3 as WIFI_PHY_STANDARD_TVWS_8MHZ and define the mapping of channel number, standard, frequency and channel width for all the channels in the band, as is done for the other standards in the existing code.

It was also necessary to define the contention window boundaries, as well as other MAC layer parameters, the band resolution, the default channel width and frequency, and a new function for configuring Orthogonal Frequency Division Multiplexing (OFDM) characteristics (e.g. short interframe space, Point Coordination Function interframe space, and device rates).

We assume that the TVWS channels in the set considered for CA are only those allowed by the GLSD for use by all the nodes in the topology considered. This would be the overlapping set of allowed channels of all the nodes at different geographical locations in that topology. Such an overlapping set may not always exist, especially if the WMN covers a large geographical area. This complication can partially be overcome by partitioning the network into clusters for which an overlapping set does exist. In this work we assume a known overlapping set exists for each topology and network size considered. This is also why we do not consider WMN sizes larger than 49 nodes in the experiments.

\subsection{Interference modelling}

The major problem we hope to solve in the research is finding an assignment of channels to links that minimises interference and so maximises $S I N R$. This means that we need to model interference and find the overall SINR for a certain CA.

Interference towards the TV transmitters is avoided through the use of a GLSD to determine allowed channels, modelled by a constrained set of channels passed to the optimisation code. More importantly for our purposes, we need to model the effects of nearby high power TV transmissions as well as other WSDs on the functioning of our TVWS WMN. We create a new subclass of SpectrumModel, constructed specifically to use channels in the TVWS band with the relevant channel widths, and create a SpectrumValue instance from that, specifying the duty cycle and period to ensure interference with our network.

To obtain the actual interference value, we use the existing InterferenceHelper code where $S I N R$ is calculated for every transmission. These snapshot $S I N R$ values are averaged over the duration of one mesh simulation run. In this way we obtain the average SINR in the network for a particular CA, network topology and interference configuration.

In ns3, frames are split into constant SINR chunks and overlapping frame chunks are considered as additional Additive White Gaussian Noise contribution to the noise [10]. Interfering signals are only considered as interference during the time when the frame chunks actually overlap with those of the wanted frame at each considered receiving node in time. Interfering signals below the energy detection threshold are not included in the computation.

\subsection{Optimisation by Simulated Annealing}

We have chosen to use the Simulated Annealing (SA) algorithm. The main issue addressed using SA is finding a link->channel mapping that maximises overall SINR in the mesh.
We also considered total throughput as a performance measure, but this is less smooth than SINR and is a derived rather than fundamental measure. Too much interference generally prevents a node from receiving packets at all, or causes it to drop its modulation and coding rate, so the throughput goes to zero or drops in steps rather than in a smooth way, as with $S I N R$, which is more suited to SA. Therefore, we do not present these results.

A single full run of the mesh simulation produces a large set of sample SINR values for traffic flow through a particular CA for a particular interference environment and network setup and topology. In the SA algorithm we need the objective function (socalled "energy" value $E$ ) to incorporate these $S I N R$ samples in a way that the desired result is the lowest cost, since SA is designed to minimise an objective function. Hence, the selected cost $E$ is based on $1 / S I N R$, shown in equation (4), where $j$ is the SA iteration number, $n$ is the number of SINR samples per node and $V$ is the number of nodes.

$$
E_{j}=\frac{1}{V} \sum_{v=1}^{V}\left[\frac{1}{n} \sum_{i=1}^{n} \frac{1}{\operatorname{SINR}_{j}(i)}(v)\right]=\frac{1}{V} \sum_{v=1}^{V} \frac{1}{\overline{S I N R_{j}}}(v)
$$

In $\mathrm{SA}$, the change in cost every iteration is used to decide whether to accept or reject the particular solution. If the new solution is better than the previous solution i.e. has a lower cost, the new solution is always accepted. However, if the new CA has a higher cost, this worse solution is accepted with a probability given by (5)

$$
h=\exp \left(-\frac{\Delta E}{k T}\right)=\exp \left(-\frac{E_{j}-E_{j-1}}{k \cdot T_{j}}\right)
$$

where

$k$ is Boltzmann's constant $\left(1.3806491023 J K^{-1}\right)$

$T_{j}$ is the temperature at iteration $j$

This is realized by selecting a random value $a$ between 0 and 1 and evaluating (6).

$$
a<h
$$

If (6) holds true, the solution is accepted. If not, the solution is rejected. If equation (5) always evaluates close to 1 , higher cost solutions will almost always be accepted and the SA algorithm will take very long converge. Conversely, if (5) always evaluates very close to 0 , almost no "worse" solutions will be accepted and the algorithm will converge prematurely on a local minimum that may be much worse than the true optimum. Therefore, a careful balance of temperature ranges, $\Delta E$ ranges as well as $k$ must be formulated to tune the algorithm appropriately. Boltzmann's constant $k$ could be omitted from this relation (or set to 1 ) in practice if it makes the probability of accepting a point extremely low, leading to converging on a local minimum. Including or leaving this constant out, or even changing its value, is part of parameter tuning required to ensure the algorithm behaves well.

The other parameter tuning that is required is the selection of the starting temperature and the temperature cooling function. A starting temperature that is too high will cause much slower convergence, as will a cooling function that decreases too slowly. On the other hand, starting with too low a temperature or a cooling function that reduces too quickly may result in converging prematurely. Starting temperature and the temperature cooling function must be adjusted in consideration of the number of iterations the algorithm is expected to run for, or that is considered acceptable. 
We considered various cooling functions in this work, e.g., exponential multiplicative cooling and logarithmic cooling [21], before finding a suitable one: linear cooling.

To summarise, selecting a combination of cost value, $k$, starting temperature, temperature cooling schedule and number of iterations is a careful balancing act, requiring experimentation to tune the values to ensure good performance of the SA algorithm.

The neighbour generation procedure we use whereby a new solution is found, is to randomly select a link, randomly select a channel, and assign the selected channel to that link, keeping the rest of the link->channel mapping unchanged.

\section{SIMULATION RESULTS}

The simulation parameters and their starting or default values are listed in Table 1.

Table 1: Parameters used in simulations with starting values

\begin{tabular}{ll}
\hline Parameter & Value \\
\hline Network size & $9-49$ nodes \\
Number of interfaces & 2 \\
Distance between grid nodes & $100 \mathrm{~m}$ \\
Channel bandwidth & $8 \mathrm{MHz}$ \\
Propagation loss model & Friis \\
Propagation delay model & ConstantSpeed \\
Packet interval & $0.01 \mathrm{~s}$ \\
Packet size & 1024 \\
SU interferer waveform power & $0.2 \mathrm{~W}$ \\
Interferer centre frequencies & $498 \mathrm{MHz}$ and 522 MHz \\
Error rate model & NistErrorRateModel \\
WMN routing algorithm & OLSR \\
\hline
\end{tabular}

For each network size, we create three different example topologies for the experiments. A topology represents a critical set of links in a WMN that require careful channel allocation and may not be the full set of links in a real network. This could represent the most constrained or bottleneck route e.g. to an Internet gateway and may not be the least hop path but might be the highest throughput or lowest delay path, even if not the most direct. This might be because of obstructions along the more direct path or lower link capacities. Examples of these generated topologies are shown in Figure 2. Topology $\mathrm{C}$ of each mesh network size is a random path.

The numbers are the node IDs. These link configurations are contrived for the purposes of illustrating how the algorithm might work to assign channels to links if the links are known, and are not meant to fully describe any real network topologies. However, they could represent the highest throughput or lowest delay paths. SU1 and SU2 represent other SU interfering radios that are not part of the WMN.

\subsection{Baseline results}

As a baseline against which our SA method can be compared, we find the mean cost value and standard deviation (SD) over 10 different random channel allocations for each of 3 different link configurations (i.e. topologies) with 0, 1 and 2 external interfering SUs respectively.

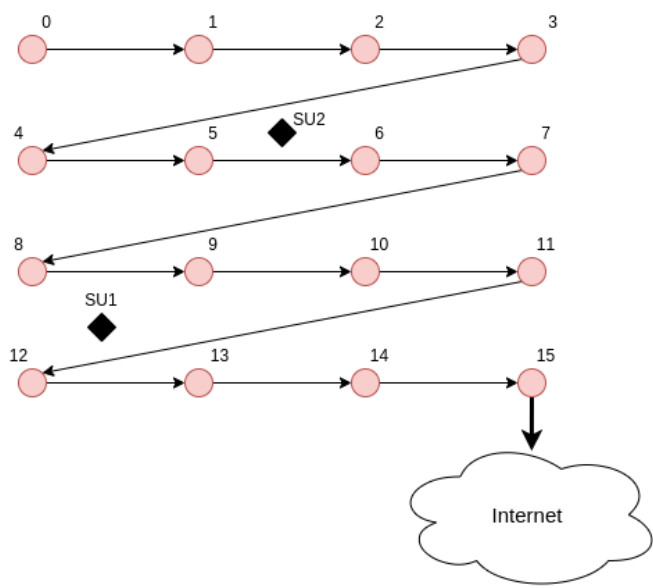

(a) Example topology $\mathrm{A}$

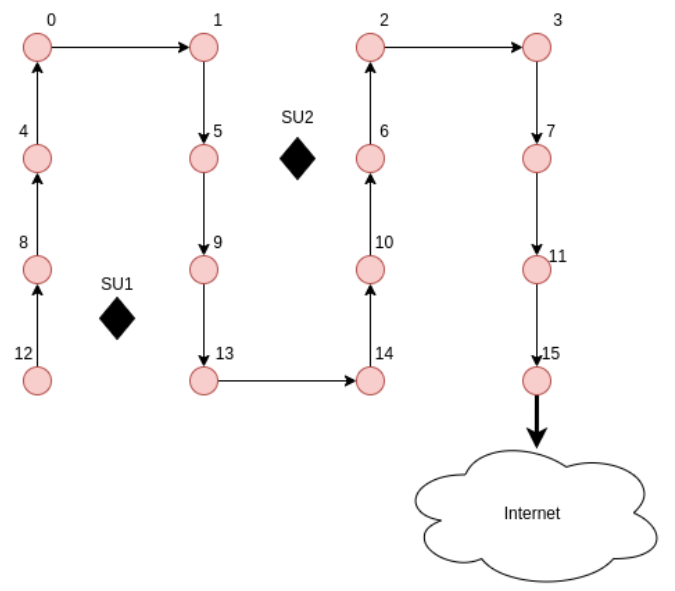

(b) Example topology B

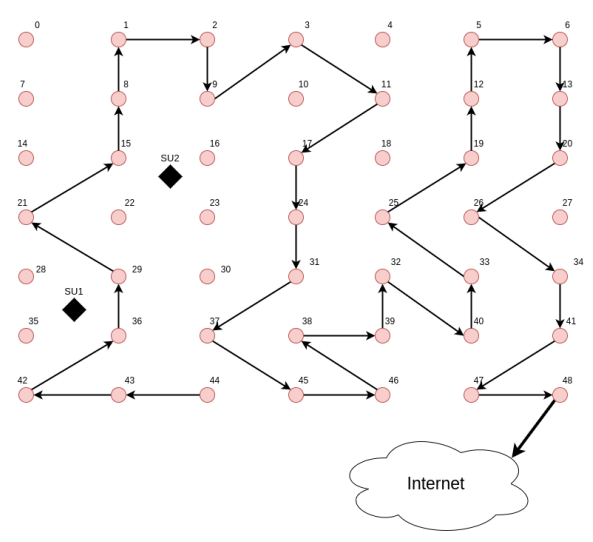

(c) Example topology $\mathrm{C}$

Figure 2: Example topologies of critical links for experiments 
Table 2: Average and standard deviation of cost values over 10 random channel allocations with no outside interference

\begin{tabular}{|c|c|c|c|c|c|c|}
\hline \multirow{3}{*}{ nodes } & \multicolumn{6}{|c|}{ Topology } \\
\hline & \multicolumn{2}{|c|}{ A } & \multicolumn{2}{|c|}{ B } & \multicolumn{2}{|c|}{ C } \\
\hline & mean & $\mathrm{SD}( \pm)$ & mean & $\mathrm{SD}( \pm)$ & mean & $\mathrm{SD}( \pm)$ \\
\hline 9 & 23.9 & 33.0 & 28.5 & 47.0 & 19.8 & 22.6 \\
\hline 16 & 23.3 & 20.8 & 21.2 & 12.6 & 11.9 & 5.0 \\
\hline 49 & 105.4 & 164.0 & 39.8 & 8.3 & 35.4 & 11.9 \\
\hline
\end{tabular}

Table 3: Average and standard deviation of cost values over 10 random channel allocations with one external interference source

\begin{tabular}{|c|c|c|c|c|c|c|}
\hline \multirow{3}{*}{ nodes } & \multicolumn{6}{|c|}{ Topology } \\
\hline & \multicolumn{2}{|c|}{ A } & \multicolumn{2}{|c|}{ B } & \multicolumn{2}{|c|}{$\mathrm{C}$} \\
\hline & mean & $\mathrm{SD}( \pm)$ & mean & $\mathrm{SD}( \pm)$ & mean & $\mathrm{SD}( \pm)$ \\
\hline 9 & 27.2 & 33.6 & 30.0 & 43.7 & 41.6 & 68.8 \\
\hline 16 & 14.6 & 7.6 & 17.7 & 11.0 & 69.6 & 142.0 \\
\hline 49 & 117.5 & 224.4 & 37.9 & 6.0 & 36.7 & 9.0 \\
\hline
\end{tabular}

Table 4: Average and standard deviation of cost values over 10 random channel allocations with two external interference sources

\begin{tabular}{|c|c|c|c|c|c|c|}
\hline \multirow{3}{*}{ nodes } & \multicolumn{6}{|c|}{ Topology } \\
\hline & \multicolumn{2}{|c|}{ A } & \multicolumn{2}{|c|}{ B } & \multicolumn{2}{|c|}{ C } \\
\hline & mean & $\mathrm{SD}( \pm)$ & mean & $\mathrm{SD}( \pm)$ & mean & $\mathrm{SD}( \pm)$ \\
\hline 9 & 24.3 & 29.3 & 20.0 & 23.8 & 18.7 & 19.9 \\
\hline 16 & 23.3 & 24.7 & 25.4 & 39.9 & 45.9 & 58.0 \\
\hline 49 & 47.9 & 25.5 & 43.9 & 8.0 & 39.5 & 7.9 \\
\hline
\end{tabular}

\subsection{Simulated Annealing results}

Initially, we attempted to run SA with a logarithmic temperature cooling function, since this is a very common cooling function. However, even over 20000 iterations, the algorithm did not converge. By inspecting the value of equation (5), we noticed the value was always very close to 1 . This means that too many poor solutions were accepted and the algorithm was not able to converge. We cannot run the algorithm for very long on the somewhat resourceconstrained nodes on which this algorithm would run in practice, so the maximum number of iterations cannot be very large. We have decided that 1000 iterations is a reasonable limit by observing running time and considering how this algorithm would be run in practice (see Section 7). We concluded that the temperature function needs to cool more quickly and changed the cooling function to a linear temperature cooling function, equation (7)

$$
T_{j}=T_{\text {start }}-\alpha \cdot j
$$

where $j$ is iteration count and $\alpha$ is a constant set to 0.02 . We also started with a lower temperature value of 20 and scaled the $1 /$ SINR values appropriately. With these adjustments the algorithm is able to converge within an acceptable 1000 iterations.

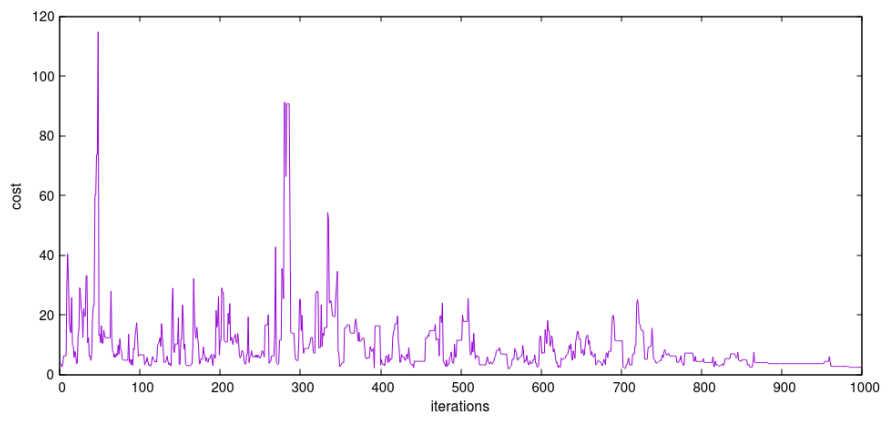

(a) 9 nodes- 0 interferers

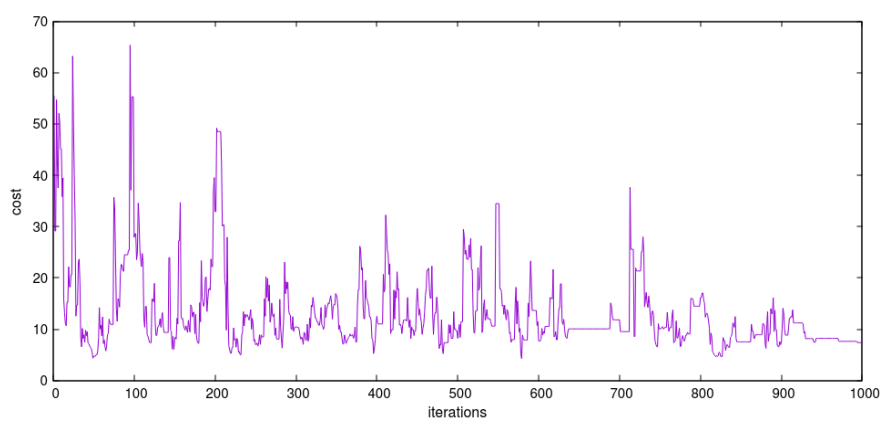

(b) 16 nodes - 1 interferer

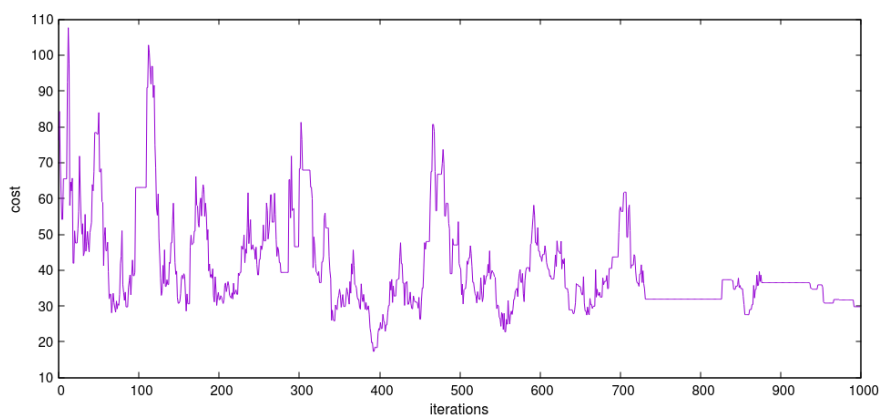

(c) 49 nodes - 2 interferers

Figure 3: The evolution of cost values over a run of Simulated Annealing with linear temperature cooling function for different WMN sizes and number of external interference sources

The plots in Figure 3 show the cost values converging towards a relatively low value within 1000 iterations for some sample runs of SA of different problem sizes. We can see how SA successfully settled on a good quality solution, even if not the overall minimum. Some resulting cost values from SA with a network size of 9 nodes and 0,1 and 2 external interference sources is given alongside the minimum and maximum values found for each experiment in Table 5 . We can see that in every case the value found was close 
to the minimum and considerably better than the worst case value (the maximum).

In Tables 6, 7 and 8, we show the average results from SA over 5 runs for each topology with 0,1 and 2 interference sources respectively and for increasing network sizes. These results must be compared with those of random channel allocations in Section 6.1. We can see in every case that SA far outperformed random CA and the standard deviation is much smaller. For 9 nodes, on average, SA yielded a $755 \%$ improvement, for 16 nodes a $571 \%$ improvement and for 49 nodes a $123 \%$ improvement, while standard deviations are many times lower. SA can guarantee good results within a small deviation. While the complexity of SA is higher, this disadvantage is negated by the improved results.

Table 5: Average of cost results (res) of SA vs the actual minimum (min) and maximum (max) for 9 nodes

\begin{tabular}{|c|c|c|c|c|c|c|c|c|}
\hline \multicolumn{9}{|c|}{ Number of interferers } \\
\hline \multicolumn{3}{|c|}{0} & \multicolumn{3}{|c|}{1} & \multicolumn{3}{|c|}{2} \\
\hline res & $\min$ & $\max$ & res & $\min$ & $\overline{\max }$ & res & $\min$ & $\overline{\max }$ \\
\hline 0.7 & 0.1 & 65.8 & 3.4 & 2.0 & 110.9 & 3.7 & 2.4 & 68.6 \\
\hline
\end{tabular}

Table 6: Average and standard deviation of cost values over 5 different $\mathrm{SA}$ runs with no external interference sources

\begin{tabular}{|c|c|c|c|c|c|c|}
\hline \multirow{3}{*}{ nodes } & \multicolumn{6}{|c|}{ Topology } \\
\hline & \multicolumn{2}{|c|}{ A } & \multicolumn{2}{|c|}{ B } & \multicolumn{2}{|c|}{ C } \\
\hline & mean & $\mathrm{SD}( \pm)$ & mean & $\mathrm{SD}( \pm)$ & mean & $\mathrm{SD}( \pm)$ \\
\hline 9 & 2.3 & 1.5 & 2.9 & 0.3 & 3.4 & 1.1 \\
\hline 16 & 2.7 & 2.8 & 4.4 & 1.6 & 3.7 & 0.9 \\
\hline 49 & 20.2 & 9.6 & 21.7 & 8.6 & 22.9 & 3.7 \\
\hline
\end{tabular}

Table 7: Average and standard deviation of cost values over 5 different $S A$ runs with one external interference source

\begin{tabular}{|c|c|c|c|c|c|c|}
\hline \multirow{3}{*}{ nodes } & \multicolumn{6}{|c|}{ Topology } \\
\hline & \multicolumn{2}{|c|}{ A } & \multicolumn{2}{|c|}{ B } & \multicolumn{2}{|c|}{ C } \\
\hline & mean & $\mathrm{SD}( \pm)$ & mean & $\mathrm{SD}( \pm)$ & mean & $\mathrm{SD}( \pm)$ \\
\hline 9 & 3.3 & 1.2 & 2.2 & 0.5 & 2.6 & 0.5 \\
\hline 16 & 6.5 & 1.3 & 4.8 & 0.5 & 3.5 & 0.6 \\
\hline 49 & 28.3 & 2.2 & 24.6 & 4.9 & 29.1 & 7.2 \\
\hline
\end{tabular}

\section{A PRACTICAL NOTE}

While this paper focuses on simulation, and practical implementation details are out of scope, for this work to be useful it needs to be implementable as well. Our method is not specific or adaptive to routing or traffic in the network, and is only dependent on the average interference and channel usage over time. This property is not expected to be fast-changing, and is only expected to change on
Table 8: Average and standard deviation of cost values over 5 different $S A$ runs with two external interference sources

\begin{tabular}{|c|c|c|c|c|c|c|}
\hline \multirow{3}{*}{ nodes } & \multicolumn{6}{|c|}{ Topology } \\
\hline & \multicolumn{2}{|c|}{ A } & \multicolumn{2}{|c|}{ B } & \multicolumn{2}{|c|}{ C } \\
\hline & mean & $\mathrm{SD}( \pm)$ & mean & $\mathrm{SD}( \pm)$ & mean & $\mathrm{SD}( \pm)$ \\
\hline 9 & 5.0 & 1.9 & 2.9 & 0.4 & 2.8 & 0.5 \\
\hline 16 & 3.7 & 3.9 & 4.5 & 1.0 & 3.9 & 0.4 \\
\hline 49 & 27.7 & 2.9 & 22.6 & 4.9 & 28.7 & 5.6 \\
\hline
\end{tabular}

the order of days in time. In a real network, we would let the nodes do spectrum sensing throughout a day whenever interfaces are not in use and then run the Simulated Annealing algorithm when the network is least busy using the already gathered samples.

We assume that the links requiring assignment are known. This could require topology discovery and/or traffic profiling to be done beforehand, so that a minimum dominating set of links is found, which are the links causing bottlenecks or with the highest traffic.

The actual spectrum sensing method by which nodes obtain SINR samples is not addressed in this work, although we assume an energy detection mechanism. A quiet period, such as proposed in IEEE 802.22 might be employed to ensure nodes can sense the effect of TV transmitters alone.

We would also require a mechanism for distributing the CA to all the nodes in the network. This would not be done by altering the MAC layer but by using a user-space messaging daemon appropriate for mesh or ad-hoc networks, such as shared-state [25] to distribute a JSON or other file-format with the CA mapping. The mechanism to ensure that the CA reaches all nodes and to coordinate channel switching is out of scope of this work, but the method of Maliwatu [20] is a possibility.

\section{FUTURE CONSIDERATIONS}

This work is only preliminary and there are plenty of avenues to explore in the future when considering DSA and alternate spectrum in WMNs and, in particular, using ns3 to simulate experiments in these areas.

Firstly, in this work we have not fully accounted for the fact that in using DSA and GLSDs, different nodes may have different sets of allowed channels. Some CAs may not be feasible for this reason and it may not be possible to find an overlapping set that is large enough to avoid interference. This must be addressed in future work.

Secondly, when comparing TVWS with Wi-Fi, it is known that the free space path loss of TVWS is lower than Wi-Fi, assuming equal gain antennas. However, actual TV antennas on the market have lower available gain than Wi-Fi antennas, whereas Wi-Fi antennas have a narrower beam width than TV antennas [14]. Gain and beam width and other more realistic antenna models should be added into the simulation in future to investigate these tradeoffs and effects of antennas on the SINR and optimal channel. The effects of other propagation loss models can also be researched.

In the real world, neither the traffic nor the channel between two node interfaces is symmetric. The channel characteristics experienced in the one direction (node $\mathrm{A}->\mathrm{B}$ ) may not be the same 
as that experienced by the signal traveling in the other direction (B->A) owing to different noise levels at the different sites [14] and different fading. This phenomenon has not been represented in the current implementation but will be a useful addition to explore in future work.

Adjacent channel interference, next-to-adjacent channel interference and spectral leakage are not modeled in ns3, since transmissions must be on the same channel to affect one another in the current model. In future, this work will be extended to model signal power leaking into neighbouring and next-to-neighbouring channels and imperfect receive signal filtering, since this is a commonly observed phenomenon and influences the channels that are truly available and optimal to use. Because of this phenomenon, the channel width also affects the choice of optimal channel. Sometimes a smaller channel width may, in fact, have better performance, even though this may seem counter-intuitive. Future work could make additions to ns 3 that take into consideration and model different channel widths and the aforementioned effects. The ideas mentioned in this section are a few of the avenues we intend to explore in future work.

\section{CONCLUSION}

In this work we had three main aims: to present a simple framework in ns3 developed for the simulation of wireless mesh networks using multiple channels and alternative spectrum, along with interference sources in that spectrum band; to present extensions to the simulator that enable the use of TVWS spectrum and creation of White Space Devices; and to present a Simulated Annealing-based channel assignment algorithm that relies on the aforementioned simulation. The latter serves as an example of how optimisation algorithms might be integrated for finding optimal channel assignments, with implementation in ns3. Our SA algorithm shows considerable improvement over random channel assignments, and we have given some pointers on the practical implementation of the method. This work is fertile ground for extensions and further research, and we have mentioned some options for future work.

\section{ACKNOWLEDGMENTS}

Thank you to all who provided input and support, and to Telkom for sponsoring the computing power for running simulations.

\section{REFERENCES}

[1] 2020. CBRS, SAS and Spectrum Sharing: The Complete Guide. https: //blinqnetworks.com/cbrs-sas-spectrum-sharing-guide/

[2] 2021. Wi-Fi Alliance ${ }^{\circledR}$ furthers Automated Frequency Coordination specification and compliance development to accelerate Wi-Fi 6E. https://www.wi-fi.org/news-events/newsroom/wi-fi-alliance-furthersautomated-frequency-coordination-specification-and

[3] A. Alsarhan, A. N. Quttoum, and Y. Kilani. 2017. Optimizing Spectrum Sharing in Wireless Mesh Network Using Cognitive Technology. Wireless Personal Communications 96, 2 (2017), 1887-1905.

[4] M. Amiri-Nezhad, M. Guerrero-Zapata, B. Bellalta, and L. Cerdà-Alabern. 2014 Simulation of multi-radio multi-channel 802.11-based mesh networks in ns-3. Eurasip fournal on Wireless Communications and Networking 2014, 1 (2014).

[5] N. Balusu, S. Pabboju, and G. Narsimha. 2019. An Intelligent Channel Assignment Approach for Minimum Interference in Wireless Mesh Networks Using Learning Automata and Genetic Algorithms. Wireless Personal Communications 106, 3 (2019), 1293-1307.

[6] Ying Yu Chen and Chien Chen. 2015. Simulated annealing for interfaceconstrained channel assignment in wireless mesh networks. Ad Hoc Networks 29 (2015), 32-44.
[7] H. Cheng, N. Xiong, A. V. Vasilakos, L.G. Tianruo Yang, and X. Zhuang. 2012. Nodes organization for channel assignment with topology preservation in multiradio wireless mesh networks. Ad Hoc Networks 10, 5 (2012), 760-773.

[8] Y. Ding and L. Xiao. 2011. Channel allocation in multi-channel wireless mesh networks. Computer Communications 34, 7 (2011), 803-815.

[9] A. B. Flores, R. E. Guerra, E. W. Knightly, P. Ecclesine, and S. Pandey. 2013. IEEE 802.11af: A standard for TV white space spectrum sharing. IEEE Communications Magazine 51, 10 (2013), 92-100.

[10] P. Fuxjaeger and S. Ruehrup. 2016. Validation of the NS-3 Interference Model for IEEE802.11 Networks. Proceedings - 2015 8th IFIP Wireless and Mobile Networking Conference, WMNC 2015 October 2015 (2016), 216-222.

[11] J. Geweke and H. Tanizaki. 2001. Bayesian estimation of state-space models using the Metropolis Hastings algorithm within Gibbs sampling. Computational statistics and data analysis 37, 2 (2001), 151-170.

[12] W. K. Hale. 1980. Frequency assignment: Theory and applications. Proc. IEEE 68, 12 (1980), 1497-1514.

[13] ICASA. 2017. DRAFT REGULATIONS ON THE USE OF TELEVISION WHITE SPACES. , 703-718 pages.

[14] D. Johnson, N. Zlobinsky, A. Lysko, M. Lamola, R. Maliwatu, and M. Densmore. 2016. Head to head battle of TV White Space and WiFi for connecting developing regions. In 8th EAI International Conference on e-Infrastructure and e-Services for Developing Countries (Africomm 2016). Ouagadougou, Burkina Faso, 186-195.

[15] M. Khalil, J. Qadir, O. Onireti, M. A. Imran, and S. Younis. 2017. Feasibility, Architecture and Cost Considerations of Using TVWS for Rural Internet Access in 5G. 2017 (2017).

[16] S. Kirkpatrick, C. D. Gelatt, and M. P. Vecchi. 1983. Optimization by simulated annealing. Science 220, 4598 (1983), 671-680.

[17] B. Ko, V. Misra, J. Padhye, and D. Rubenstein. 2007. Distributed Channel Assignment in Multi-Radio 802.11 Mesh Networks. In Wireless Communications and Networking Conference.

[18] Natallia Kokash. 2005. An introduction to heuristic algorithms. (2005).

[19] D. J. Leith, P. Clifford, V. Badarla, and D. Malone. 2012. WLAN channel selection without communication. Computer Networks 56, 4 (2012), 1424-1441.

[20] R. Maliwatu. 2020. A new connectivity strategy for Wireless Mesh Networks using Dynamic Spectrum Access). Ph.D. Dissertation. University of Cape Town, Rondebosch, Cape Town, South Africa. Advisor(s) Johnson, D. L.

[21] Y. Nourani and B. Andresen. 1998. A comparison of simulated annealing cooling strategies. Journal of Physics A: Mathematical and General 31, 41 (1998), 83738385.

[22] Tina Pelkey. 2020. FCC Adopts New Rules for the 6 GHz Band, Unleashing 1.200 Megahertz of Spectrum for Unlicensed Use.

[23] A. Raniwala, K. Gopalan, and T. Chiueh. 2004. Centralized channel assignment and routing algorithms for multi-channel wireless mesh networks. ACM SIGMOBILE Mobile Computing and Communications Review 8, 2 (2004), 50.

[24] K. K. Ronoh, G. Kamucha, T. Olwal, and T. Omwansa. 2019. A survey of resource allocation in TV White Space networks. Journal of Communications 14, 12 (2019), $1180-1190$.

[25] shared-state 2021. shared-state. Retrieved May 15, 2021 from https://github.com/ libremesh/lime-packages/tree/master/packages/shared-state

[26] T. Shen, S. Toledo, Z. Gu, S. Zhang, Y. Wang, and M. Song. 2018. Where to rendezvous? Preferring quiet channels in cognitive radio networks. MSWiM 2018 - Proceedings of the 21st ACM International Conference on Modeling, Analysis and Simulation of Wireless and Mobile Systems (2018), 325-332.

[27] M. Shojafar, Z. Pooranian, M. Shojafar, and A. Abraham. 2014. LLLA: New Efficient Channel Assignment Method in Wireless Mesh Networks. In Advances in Intelligent Systems and Computing, Vol. 237. 143-152.

[28] S. Sridhar, J. Guo, and S. Jha. 2009. Channel assignment in multi-radio wireless mesh networks : A graph-theoretic approach. In 2009 First International Communication Systems and Networks and Workshops. 1-10.

[29] R. Struzak and D. Wiecek. 2013. TV White Spaces: A Pragmatic Approach (first ed.). ICTP-The Abdus Salam International Centre for Theoretical Physics T/ICT4D Lab, Trieste. 19-34 pages.

[30] A. P. Subramanian, H. Gupta, and S. R. Das. 2007. Minimum Interference Channel Assignment in Multi-Radio Wireless Mesh Networks. In 2007 th Annual IEEE Communications Society Conference on Sensor, Mesh and Ad Hoc Communications and Networks. 481-490.

[31] M. Zennaro, E. Pietrosemoli, A. Arcia-moret, J. Pinifolo, C. Wang, and S. Song. 2013. TV White Spaces, I Presume? The Quest for TVWS in Malawi and Zambia. Proceedings of the Sixth International Conference on Information and Communication Technologies and Development Full Papers - ICTD '13 - volume 1 (2013), $12-15$. 